\title{
A PARTITION THEOREM FOR EUCLIDEAN $n$-SPACE ${ }^{1}$
}

\author{
HANS SAMELSON, R. M. THRALL AND OSCAR WESLER
}

Let $V^{n}$ be an $n$-dimensional vector space over the reals. Let $\xi_{1}, \cdots, \xi_{n}, \eta_{1}, \cdots, \eta_{n}$ be $2 n$ vectors in $V^{n}$ such that every sequence of vectors $\left\{\alpha_{1}, \cdots, \alpha_{n}\right\}$, where $\alpha_{i}$ is either $\xi_{i}$ or $\eta_{i}$, is a linearly independent set. Let $\left\langle\alpha_{1}, \cdots, \alpha_{n}\right\rangle$ denote the cone spanned by these $\alpha$ 's, i.e. the set of all linear combinations of the $\alpha_{i}$ with non-negative coefficients. The $2^{n}$ cones in $V^{n}$ spanned by the $2^{n}$ such sequences of $\alpha$ 's will be said to partition $V^{n}$ if their union is all of $V^{n}$ and if the intersection of every pair of distinct cones $\left\langle\alpha_{1}, \cdots, \alpha_{n}\right\rangle,\left\langle\alpha_{1}^{\prime}, \cdots, \alpha_{n}^{\prime}\right\rangle$ is precisely the lower-dimensional cone ("face," "edge," etc.) spanned by the common vectors, i.e. by those $\alpha_{i}$ for which $\alpha_{i}=\alpha_{i}^{\prime}$, or $\{0\}$ if there are no such $\alpha_{i}$. We may and shall assume that $V^{n}$ is the usual Euclidean space $E^{n}$ of ordered $n$-tuples of reals. The most familiar example of a partition is the one with the unit vectors $(1,0,0, \cdots, 0)$, $(0,1,0, \cdots, 0), \cdots,(0, \cdots, 0,0,1)$ in the role of the $\xi$ 's, and their negatives in the role of the $\eta$ 's. The partitioning cones are then the $2^{n}$ orthants into which the rectangular coordinate axes divide the space. In this paper, a simple necessary and sufficient condition that the $\xi$ 's and $\eta$ 's provide a partition is given; the result is reinterpreted in the language of matrices.

Let the orientation of an ordered set of vectors $\left\{\alpha_{1}, \alpha_{2}, \cdots, \alpha_{n}\right\}$ be given as usual by the sign of the determinant, sgn $\operatorname{det}\left(\alpha_{1}, \cdots, \alpha_{n}\right)$. Any $n-1$ of the $\alpha$ 's determine a hyperplane, separating $E^{n}$ into the two half-spaces lying on opposite sides of the hyperplane. For the sake of simplicity in the statement of the theorem, we assume without loss of generality that the ordered set $\left\{\xi_{1}, \xi_{2}, \cdots, \xi_{n}\right\}$ is positively oriented.

THEOREM. The $2^{n}$ cones $\left\langle\alpha_{1}, \cdots, \alpha_{n}\right\rangle$ partition $E^{n}$ if and only if sgn det $\left(\alpha_{1}, \cdots, \alpha_{n}\right)=(-1)^{\text {s }}$ where $s=$ the number of $\eta$ 's among the $\alpha$ 's; equivalently, if and only if, with respect to every choice of $n-1 \alpha$ 's, the $\xi$ and $\eta$ for the omitted index are separated by the hyperplane determined by these $\alpha$ 's.

Received by the editors March 15, 1958.

1 The research for this paper was supported, in part, by an Air Force Contract with the Engineering Research Institute of the University of Michigan (AF29(601)467 ). This theorem was stimulated by a problem in structural engineering which was communicated to the authors by Dr. Glen V. Berg of the Department of Civil Engineering of the University of Michigan. 
Proof. The equivalence of the determinant and separation condition is obvious; we shall give the proof of the theorem using the geometric language of half-spaces and separating hyperplanes wherever possible.

Necessity is immediate, for if the $\xi$ and $\eta$ of any index, say the $j$ th, were to lie in the same half-space relative to any choice of vectors $\alpha_{1}, \cdots, \alpha_{j-1}, \alpha_{j+1}, \cdots, \alpha_{n}$ for the remaining indices, then the two cones $\left\langle\alpha_{1}, \cdots, \alpha_{j-1}, \xi_{j}, \alpha_{j+1}, \cdots, \alpha_{n}\right\rangle$ and $\left\langle\alpha_{1}, \cdots, \alpha_{j-1}, \eta_{j}\right.$, $\left.\alpha_{j+1}, \cdots, \alpha_{n}\right\rangle$ would clearly have an $n$-dimensional intersection, contradicting partition.

To prove sufficiency, consider first the union of the $2^{n}$ cones, which is a closed set in $E^{n}$. Its complement, if not empty, is an open set, whose boundary is made up of $(n-1)$-dimensional pieces of certain $(n-1)$-dimensional faces of certain of the cones. But no $(n-1)$ dimensional face can contribute such pieces to the boundary, for the fact that the omitted $\xi$ and $\eta$ lie in opposite half-spaces clearly places the relative interior of a face in the interior of the union of the two cones spanned by the face and the omitted $\xi$ and $\eta$ respectively. Hence the complement is empty, and the union of the $2^{n}$ cones must be all of $E^{n}$.

It remains to prove the intersection property, that if two sequences $\left\{\alpha_{1}, \cdots, \alpha_{n}\right\},\left\{\alpha_{1}^{\prime}, \cdots, \alpha_{n}^{\prime}\right\}$ have exactly $r$ vectors in common, then the intersection of the associated cones is the $r$-cone spanned by the $r$ vectors. The proof is by finite induction on $n-r$. For $n-r=0$ the statement is trivially true; for $n-r=1$ it follows at once from the separation condition. Suppose the property true for $n-r=k$, and consider two cones $L$ and $M$ whose spanning vector sets have exactly $r-1=n-k-1$ vectors in common. We may assume that the two sequences are $\left\{\xi_{1}, \cdots, \xi_{n}\right\}$ and $\left\{\xi_{1}, \cdots, \xi_{r-1}, \eta_{r}, \cdots, \eta_{n}\right\}$ respectively. Suppose there exists a vector $\delta$ in the intersection of the two cones but not in $\left\langle\xi_{1}, \cdots, \xi_{r-1}\right\rangle$. We note that $\delta$ cannot lie in a face of $M$ opposite to an $\eta_{i}$ with $r \leqq i \leqq n$; if it did, it would contradict the induction assumption for the cones $L$ and $M^{\prime}$, where $M^{\prime}$ is obtained from $M$ by replacing this $\eta_{i}$ by $\xi_{i}$ (so that the vector sequences of $L$ and $\bar{M}^{\prime}$ have $r$ terms in common). By adding an element of $\left\langle\xi_{1}, \cdots, \xi_{r-1}\right\rangle$ if necessary, we may assume that $\delta$ lies in the interior of $M$. The vector $\xi_{r}$ has a unique representation $\xi_{r}^{\prime}+\xi_{r}^{\prime \prime}$, with $\xi_{r}^{\prime}=\sum_{i=1}^{r-1} a_{i} \xi_{i}, \xi_{r}^{\prime \prime}=\sum_{i=r}^{n} a_{i} \eta_{i}$. Let $\zeta_{r}$ be any element of $\left\langle\xi_{1}, \cdots, \xi_{r-1}\right\rangle$ such that $\xi_{r}^{\prime}+\zeta_{r}$ lies in the interior of $\left\langle\xi_{1}, \cdots, \xi_{r-1}\right\rangle$. We form $\delta_{t}=\delta+t\left(\xi_{r}+\zeta_{r}\right)$, for $t \geqq 0$. Clearly $\delta_{t}$ belongs to $L$ for all such $t$, but does not belong to $\left\langle\xi_{1}, \cdots, \xi_{r-1}\right\rangle$. Furthermore, $\delta_{t}$ will never lie in a face of $M$ opposite one of the $\xi_{i}, 1 \leqq i \leqq r-1$, by the choice of $\zeta_{r}$. On 
the other hand, for large $t$-values $\delta_{t}$ and $\eta_{r}$ lie on opposite sides of the hyperplane spanned by $\left\{\xi_{1}, \cdots, \xi_{r-1}, \eta_{r+1}, \cdots, \eta_{n}\right\}$ because of the basic separation hypothesis on $\xi_{r}$ and $\eta_{r}$. This means that for large $t$-values $\delta_{t}$ is outside of the cone $M$. There exists then a positive $t_{0}$ such that $\delta_{t_{0}}$ lies in a face of $M$. As we just saw, this must be a face opposite to one of the $\eta_{i}, r \leqq i \leqq n$. But by the argument that was applied to $\delta$ in the beginning, this yields a contradiction to the induction assumption, so that the intersection property must hold and the theorem is proved.

It is instructive as well as useful in facilitating applications and for computational purposes to view the foregoing matter in the light of matrices. Accordingly, let $A$ and $B$ be the square matrices whose columns are, respectively, the components of the $\xi_{i}$ and $\eta_{i}$. We will say that the matrices $A$ and $B$ give a partition whenever their columns do. The partitioning condition on the $\xi$ 's and $\eta$ 's is clearly a centro-affine invariant, that is to say invariant under the full linear group of all nonsingular linear transformations on $E^{n}$; under a nonsingular linear transformation with matrix $P$, the matrices $A$ and $B$ are replaced by $P A$ and $P B$, and the matrices $P A$ and $P B$ give a partition if and only if the matrices $A$ and $B$ do. Further, if sgn det $P$ $=1$, then the positive orientation of the first set of vectors is preserved. Under the assumption that matrices $A$ and $B$ give a partition and sgn $\operatorname{det} A=1$, it follows from our main theorem that sgn $\operatorname{det} B$ $=(-1)^{n}$, or that $\operatorname{sgn} \operatorname{det}(-B)=1$. Hence $\operatorname{sgn} \operatorname{det}\left(-B^{-1}\right)=1$, so that the pair $C,-I$ with $C=-B^{-1} A$ again gives a partition, with the first set of vectors in a positive orientation. (As a matter of fact, $C$ will be positively oriented even when $A$ is not, as may be verified immediately.) Now let $D$ be obtained from $C$ by replacing some $h$ columns of $C$ by the corresponding columns of $-I$. Then $\operatorname{det} D$ $=(-1)^{h} \operatorname{det} C^{\prime}$ where $C^{\prime}$ is the principal submatrix of $C$ obtained by deleting the given $h$ columns and corresponding rows. By the main theorem, however, sgn $\operatorname{det} D=(-1)^{h}$, so that sgn $\operatorname{det} C^{\prime}=1$. Putting the argument together, we conclude with the simple condition that two matrices $A$ and $B$, equivalently the matrices $C=-B^{-1} A$ and $-I$, give a partition if and only if every principal minor of $C$ is positive.

Although many of the above arguments go through unchanged, the proper formulation of the problem in the infinite dimensional case is not clear. Presumably, a linear topological space would provide the proper setting for it.

UNIVERSITY OF MICHIGAN 